\title{
UNVEILING A POPULATION OF GALAXIES HARBORING LOW-MASS BLACK HOLES WITH X-RAYS
}

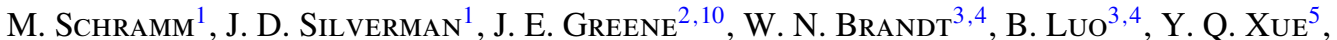

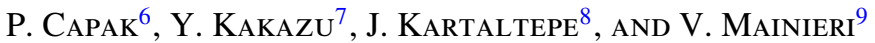 \\ ${ }^{1}$ Kavli Institute for the Physics and Mathematics of the Universe (WPI), Todai Institutes for Advanced Study, the University of Tokyo, \\ Kashiwanoha 5-1-5, Kashiwa-shi, Chiba 277-8568, Japan; malte.schramm@ipmu.jp \\ 2 Department of Astrophysical Science, Princeton University, Princeton, NJ 08544, USA \\ ${ }^{3}$ Department of Astronomy and Astrophysics, Pennsylvania State University, University Park, PA 16802, USA \\ ${ }^{4}$ Institute for Gravitation and the Cosmos, Pennsylvania State University, University Park, PA 16802, USA \\ ${ }^{5}$ Key Laboratory for Research in Galaxies and Cosmology, Department of Astronomy, University of Science and Technology of China, \\ Chinese Academy of Sciences, Hefei, Anhui 230026, China \\ ${ }^{6}$ Spitzer Science Center, 314-6 California Institute of Technology, 1201 East California Boulevard, Pasadena, CA 91125, USA \\ ${ }^{7}$ Department of Astronomy, California Institute of Technology, Pasadena, CA 91125, USA \\ ${ }^{8}$ National Optical Astronomy Observatory, 950 North Cherry Avenue, Tucson, AZ 85719, USA \\ ${ }^{9}$ European Southern Observatory, Karl-Schwarzschild-Strasse 2, Garching, D-85748, Germany \\ Received 2013 February 22; accepted 2013 May 10; published 2013 August 5
}

\begin{abstract}
We report the discovery of three low-mass black hole (BH) candidates residing in the centers of low-mass galaxies at $z<0.3$ in the Chandra Deep Field-South Survey. These BHs are initially identified as candidate active galactic nuclei based on their X-ray emission in deep Chandra observations. Multi-wavelength observations are used to strengthen our claim that such emission is powered by an accreting supermassive $\mathrm{BH}$. While the X-ray luminosities are low at $L_{X} \sim 10^{40} \mathrm{erg} \mathrm{s}^{-1}$ (and variable in one case), we argue that they are unlikely to be attributed to star formation based on H $\alpha$ or UV fluxes. Optical spectroscopy from Keck and the VLT allows us to (1) measure accurate redshifts, (2) confirm their low stellar host mass, (3) investigate the source(s) of photo-ionization, and (4) estimate extinction. With stellar masses of $M_{*}<3 \times 10^{9} M_{\odot}$ determined from Hubble Space Telescope/Advanced Camera for Surveys imaging, the host galaxies are among the lowest mass systems known to host actively accreting BHs. We estimate $\mathrm{BH}$ masses $M_{\mathrm{BH}} \sim 2 \times 10^{5} M_{\odot}$ based on scaling relations between $\mathrm{BH}$ mass and host properties for more luminous systems. In one case, a broad component of the $\mathrm{H} \alpha$ emission-line profile is detected, thus providing a virial mass estimate. BHs in such low-mass galaxies are of considerable interest as the low-redshift analogs to the seeds of the most massive BHs at high redshift which have remained largely elusive to date. Our study highlights the power of deep X-ray surveys to uncover such low-mass systems.
\end{abstract}

Key words: galaxies: active - X-rays: galaxies

\section{INTRODUCTION}

At the present time, nearly all massive galaxies contain supermassive $\left(10^{6}-10^{9} M_{\odot}\right)$ black holes $(\mathrm{BHs})$ at their center. The formation mechanism of the seeds for these BHs is not known. They may have formed from the death of the first, massive stars (e.g., Li et al. 2007; Ohkubo et al. 2009; Bromm \& Yoshida 2011), from the direct collapse of gas clouds (e.g., Koushiappas et al. 2004), or in the centers of dense stellar clusters (e.g., Devecchi \& Volonteri 2009). Whatever the mechanism, we know that in some cases the seed BHs were able to gain mass quickly to form massive quasars at $z \sim 6$ (e.g., Fan et al. 2001). Additional insight into the formation of the first BHs comes from looking for the least massive BHs in the universe today. These are, in some sense, "leftover" seed BHs. A study of their number density, mass distribution, and host-galaxy properties can in principle isolate the primary seed formation mechanism (e.g., Volonteri \& Natarajan 2009; Van Wassenhove et al. 2012).

Unfortunately, low-mass BHs $\left(M_{\mathrm{BH}}<10^{6} M_{\odot}\right)$ are difficult to study since they tend to live in faint galaxies. Even when active, the BHs are faint, rarely reaching their Eddington luminosity $\left(L_{\text {Edd }} \sim 10^{43} \mathrm{erg} \mathrm{s}^{-1}\right.$ for a $\left.\sim 10^{5} M_{\odot} \mathrm{BH}\right)$. The first indication of a population of low-mass BHs in late-type galaxies came from

\footnotetext{
${ }^{10}$ Alfred P. Sloan Fellow.
}

the active galactic nucleus (AGN) discovered in NGC 4395 (Filipenko \& Sargent 1989), which is thought to harbor a $\sim 10^{5} M_{\odot}$ BH (Filippenko \& Ho 2003; Peterson et al. 2005). The rediscovery of the AGN in POX 52, also thought to harbor a $\sim 10^{5} M_{\odot}$ BH (Barth et al. 2004), inspired a number of more systematic searches for BHs in this low-mass range. Systematic searches through the Sloan Digital Sky Survey (SDSS) have uncovered more than 100 BHs with $M_{\mathrm{BH}}<10^{6} M_{\odot}$ (Greene \& Ho 2004; Greene \& Ho 2007a; Dong et al. 2007, 2012). Deeper surveys of late-type spirals with optical and mid-infrared spectroscopy have uncovered a handful of additional examples (Shields et al. 2007; Satyapal et al. 2007, 2009; Desroches et al. 2009; Reines et al. 2011, 2012). We are beginning to study the accretion properties (Greene et al. 2006; Greene \& Ho 2007a; Desroches et al. 2009; Wrobel et al. 2008; Miniutti et al. 2007; Dong et al. 2012; Ludwig et al. 2012) and the distribution of host properties for these samples (Barth et al. 2005; Greene et al. 2008; Xiao et al. 2011; Jiang et al. 2011a). On the other hand, because of severe bias in the optical selection of these objects, it is prohibitive to calculate a robust space density for them (see review in Greene 2012).

Alternate search strategies are needed that circumvent strong optical bias toward luminous host galaxies and high-Eddingtonratio sources. Techniques using X-rays or mid-infrared spectroscopy have utilized very small samples to date (Dewangan et al. 2008; Desroches et al. 2009; Satyapal et al. 2009; Gallo 
et al. 2010; Leipski et al. 2012; Ho et al. 2012; Terashima et al. 2012; Araya Salvo et al. 2012; Secrest et al. 2012). On the other hand, very deep X-ray surveys now exist that reach depths capable of probing the intermediate-mass regime for BHs. For instance, the central area of the Chandra Deep Field-South Survey reaches depths (up to $4 \mathrm{Ms}$ ) capable of uncovering $\sim 10^{5}-10^{6} M_{\odot}$ BHs with $L_{\text {bol }} / L_{\text {Edd }}$ as low as $10^{-3}$ out to $z \approx 1$ (Babić et al. 2007). So far, most studies of the AGN population in these deep-survey fields have focused on either the high-redshift luminous population or those hidden by obscuration. Recently, Xue et al. (2012) find that there is a significant fraction of relatively low mass galaxies that host highly obscured AGNs at $z \sim 1-3$, which contribute to the unresolved $6-8 \mathrm{keV}$ cosmic $\mathrm{X}$-ray background. These surveys enable searches for accreting BHs in small stellar mass systems that are not biased by host-galaxy mass. Furthermore, such surveys have remarkable multiwavelength data that greatly facilitate our understanding of the building blocks of the more massive quasars and their host galaxies.

We use X-ray observations from the Chandra Deep FieldSouth Survey and its extended coverage to search for faint AGNs within the low-mass $\left(\sim 10^{8}-10^{9} M_{\odot}\right)$ galaxy population. These are galaxies most likely to harbor low-mass BHs. We select candidates with high X-ray luminosities compared to our expectations based on their optical luminosities and star formation rates (SFRs). In this exploratory work, we highlight three candidates with $0.1<z<0.3$, for which we also have high-quality optical spectroscopy. From the spectra, we seek additional signs of AGN activity from the strong line ratios, estimate the level of dust extinction, and place some limits on the SFRs. Using high-resolution imaging from Hubble Space Telescope/Advanced Camera for Surveys (HST/ACS), we present properties of the host galaxies. A follow-up study (M. Schramm et al., in preparation) will present the full population of AGNs associated with low-mass galaxies up to $z \sim 1$.

\section{DATA AND SAMPLE SELECTION}

We first select a sample of low-mass galaxies using the GEMS survey (Rix et al. 2004) in the Extended Chandra Deep FieldSouth (E-CDF-S) field that provides $H S T /$ ACS imaging over $0.3 \mathrm{deg}^{2}$. Photometric redshifts are provided by the MUSYC survey (Cardamone et al. 2010) that are based on 32 bands of optical and infrared imaging. Due to the wealth of spectroscopic programs in the E-CDF-S (e.g., Szokoly et al. 2004; Vanzella et al. 2008; Popesso et al. 2009; Treister et al. 2009; Silverman et al. 2010; Cooper et al. 2012), we are able to replace photometric redshifts with spectroscopic values if available. We start by selecting galaxies with $z<1$ such that one of the two HST filters falls above the $4000 \AA$ break for sensitivity to older stellar populations. Stellar masses are determined from the galaxy absolute magnitude $M_{V}$ and rest-frame color $B-V$ as measured from HST imaging, following the prescription of Bell et al. (2003). Our stellar mass estimates match well with those from the MUSYC survey (Cardamone et al. 2010) as derived from broadband spectral energy distribution (SED) fitting, for galaxies with secure spectroscopic redshifts that are in agreement with the photometric redshifts.

In this sample, there are $\sim 5200$ galaxies with $M_{*}<3 \times$ $10^{9} M_{\odot}$. The galaxy cutoff mass we choose is somewhat arbitrary, but does focus on a stellar-mass regime where only a few AGNs are already known (see, e.g., Figure 1 of Xue et al. 2010; note the $3 \times 10^{9} M_{\odot}$ cutoff line in their Figure 1).

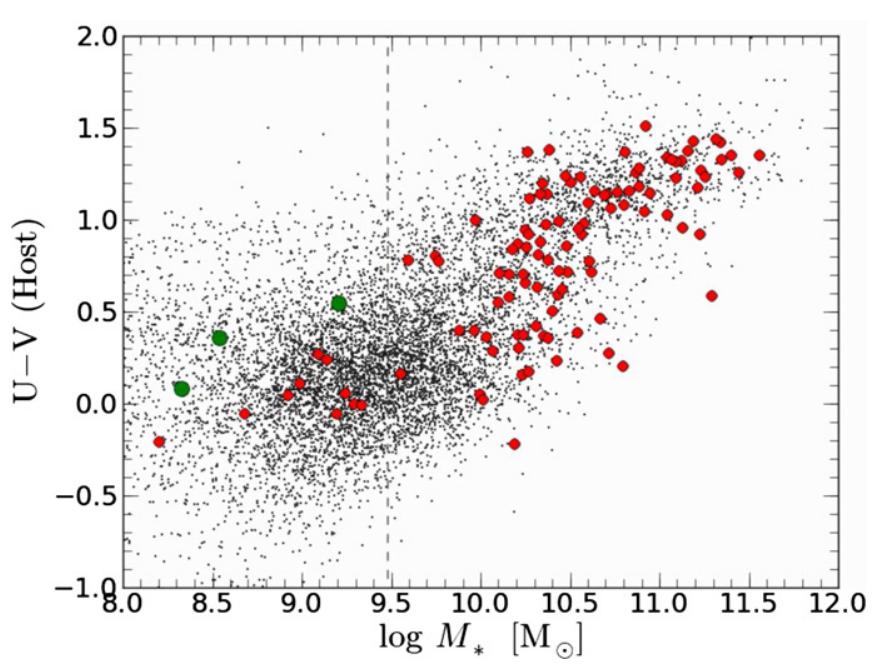

Figure 1. Rest-frame $U-V$ as a function of stellar mass for galaxies $(z<1)$ in the GEMS survey. All X-ray sources with $L_{X}<10^{44} \mathrm{erg} \mathrm{s}^{-1}$ and $z_{\text {spec }}<1$ are shown in red, while those of interest for this study at $z<0.3$ are marked in green. The dashed line indicates our selection criterion of $M_{*}<3 \times 10^{9} M_{\odot}$.

Specifically, low-mass BHs found through optical selection using the SDSS (Greene \& Ho 2007b) are typically found in higher-mass galaxies with stellar masses of a few times $10^{9}-10^{10} M_{\odot}$ (see also Dong et al. 2012). The stellar-mass regime below $\sim 10^{9} M_{\odot}$ has yielded essentially no AGNs in optically selected searches (Ho et al. 1997; Kauffmann et al. 2003; Barth et al. 2008). Also, based on a naive extrapolation of the BH-to-bulge mass relation of Haering \& Rix (2004), these galaxies are likely to harbor a $\mathrm{BH}$ with $M_{\mathrm{BH}}<2 \times 10^{6} M_{\odot}$. Finally, the upper limit on the stellar mass of the parent galaxy population focuses our search for low-mass BHs at the lowmass end of the blue cloud, a region of parameter space in the color-mass plane (see Figure 1), where galaxies are likely to be forming stars rapidly.

Multi-wavelength catalogs of X-ray sources in the CDF-S (Young et al. 2010; Silverman et al. 2010; Xue et al. 2011) provide robust matches to optical counterparts, including lowmass galaxies, based upon a likelihood-ratio matching routine. We find 27 galaxies with $z<1, M_{*}<3 \times 10^{9} M_{\odot}$, and an $\mathrm{X}$-ray detection. We note that all X-ray sources have a wavdetect false-positive probability of $10^{-8}$. Of these 27 galaxies, we concentrate here on the three galaxies (out of a parent population of 2100) with $z<0.3$ and existing optical spectra and a high ratio of $\mathrm{X}$-ray to optical luminosity. At these redshifts our spectra cover four strong optical emission lines: $\mathrm{H} \beta$, [O III] $\lambda \lambda 4959,5007, \mathrm{H} \alpha$, and [N II] $\lambda \lambda 6548,6584$, which will be used for further classification and analysis.

In this paper, we present three candidate AGNs in lowmass galaxies, selected to have a high ratio of X-ray to optical luminosity as detailed below. If confirmed, these galaxies would be among the lowest-mass host galaxies known (see also Barth et al. 2008; Reines et al. 2011). Two of them (XID-476, XID-231) are detected in the shallower E-CDF-S coverage (Lehmer et al. 2005) and the third (XID-312) within the central 4 Ms area (Xue et al. 2011) with more than 100 counts in the full band $(0.5-8 \mathrm{keV})$. In Figure 2, we show postagestamp HST/ACS images of the three low-mass galaxies while indicating the $\mathrm{X}$-ray centroid and an effective search radius for an optical counterpart that demonstrates the likelihood of these associations. In the remainder of this paper, we combine the HST imaging (Figure 2), the optical spectroscopy from 

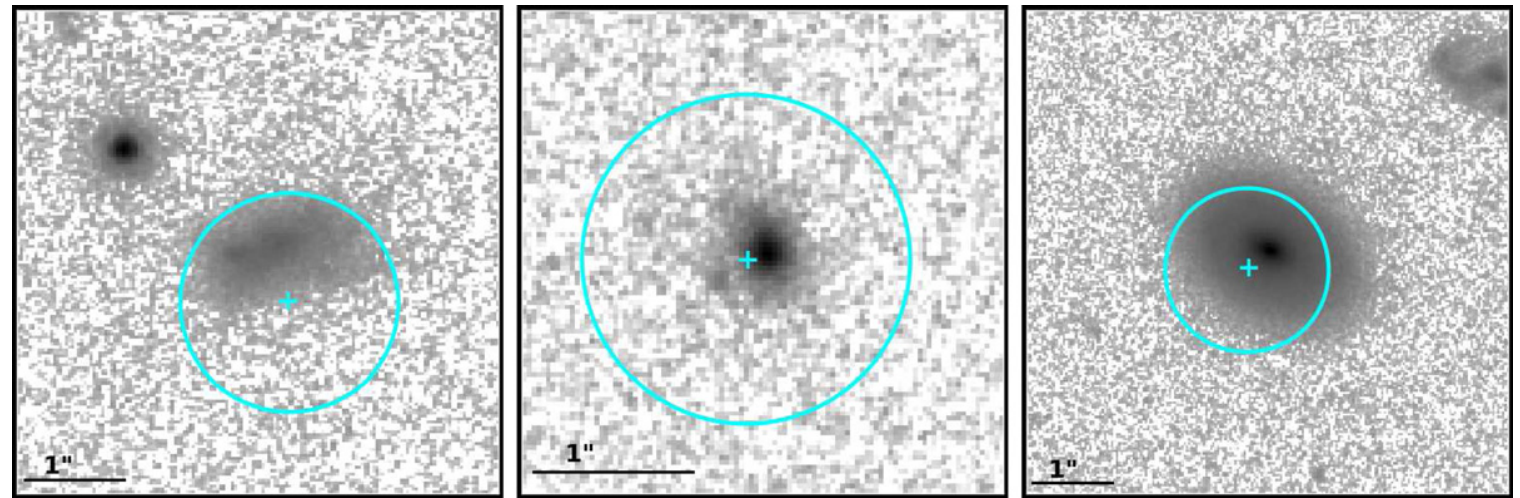

Figure 2. HST/ACS images of three low-mass galaxies determined to be the optical counterparts of the Chandra detections (left: 231; middle: 476; right: 312). A blue cross marks the centroid position of the X-ray detections. A circle indicates an effective search radius of $1^{\prime \prime}$.

Keck/DEIMOS (Silverman et al. 2010) and VLT/VIMOS (Szokoly et al. 2004) (Figure 3), and the broadband SED to argue that each of these sources is a viable candidate to host a low-mass AGN (Section 3).

\section{DISENTANGLING X-RAY EMISSION FROM AGNs AND NORMAL GALAXIES}

We present our case that these three X-ray-detected galaxies are most naturally explained as being powered by accretion onto a massive $\mathrm{BH}$. There are many other processes that lead to X-ray emission from galaxies, including an unresolved contribution of low-mass and high-mass X-ray binaries or thermal emission from hot gas (e.g., Fabbiano 2006). In particular, the high-mass $\mathrm{X}$-ray binary population is likely to contribute significantly to those galaxies with high SFRs. Fortunately, there are many studies (e.g., Ranalli et al. 2003; O'Sullivan et al. 2003; Lehmer et al. 2010; Boroson et al. 2011) of X-ray emission from inactive galaxies (with no AGN) that enable us to determine whether the level of X-ray emission is characteristically higher than that expected for galaxies of a given luminosity (i.e., stellar mass) and SFR.

We first show that our candidates have excess X-ray emission compared to that expected from early-type galaxies based on their optical continuum luminosities and then we show that the sources have even more anomalous X-ray luminosity given their inferred SFRs. In Figure 4, we plot the distribution of X-ray luminosity as a function of $B$-band luminosity. The $1 \sigma$ region of parameter space where $\mathrm{X}$-ray emission is characteristic of earlytype galaxies (O'Sullivan et al. 2003) is shaded. For reference, we also include all galaxies with X-ray detections $\left(L_{X}<\right.$ $10^{44} \mathrm{erg} \mathrm{s}^{-1}$ ) and $z<1 \mathrm{in} \mathrm{red.} \mathrm{The} \mathrm{dashed} \mathrm{line} \mathrm{gives} \mathrm{the} 1 \sigma$ upper boundary for star-forming galaxies (Lehmer et al. 2010). We show the location of our three low-mass galaxies at $z<0.3$ that are X-ray emitters (green points). Although XID-312 is just above our selection criteria in the $L_{X}-L_{B}$ plane, the galaxy has been reported to be X-ray variable in the recent study by Young et al. (2012), who concluded that the observed variability cannot be due to binary populations or ultraluminous sources but rather is best explained by an accreting black hole. Its X-ray hardness ratio, defined as HR $=(H-S) /(H+S)$, where $S$ and $H$ are the soft $(0.5-2 \mathrm{keV})$ and hard $(2-8 \mathrm{keV})$ band net counts, is HR $=-0.2$ (Xue et al. 2011). The observed hardness ratio suggests some absorption by neutral gas with a density of about $N_{\mathrm{H}} \sim 10^{22} \mathrm{~cm}^{-2}$ (see Figure 9 in Silverman et al. 2005). There are 22 low-mass $\left(M_{*}<3 \times 10^{9} M_{\odot}\right)$ galaxies at $z<1$ that have an $L_{X}$-to- $L_{B}$ ratio more than $1 \sigma$ away from
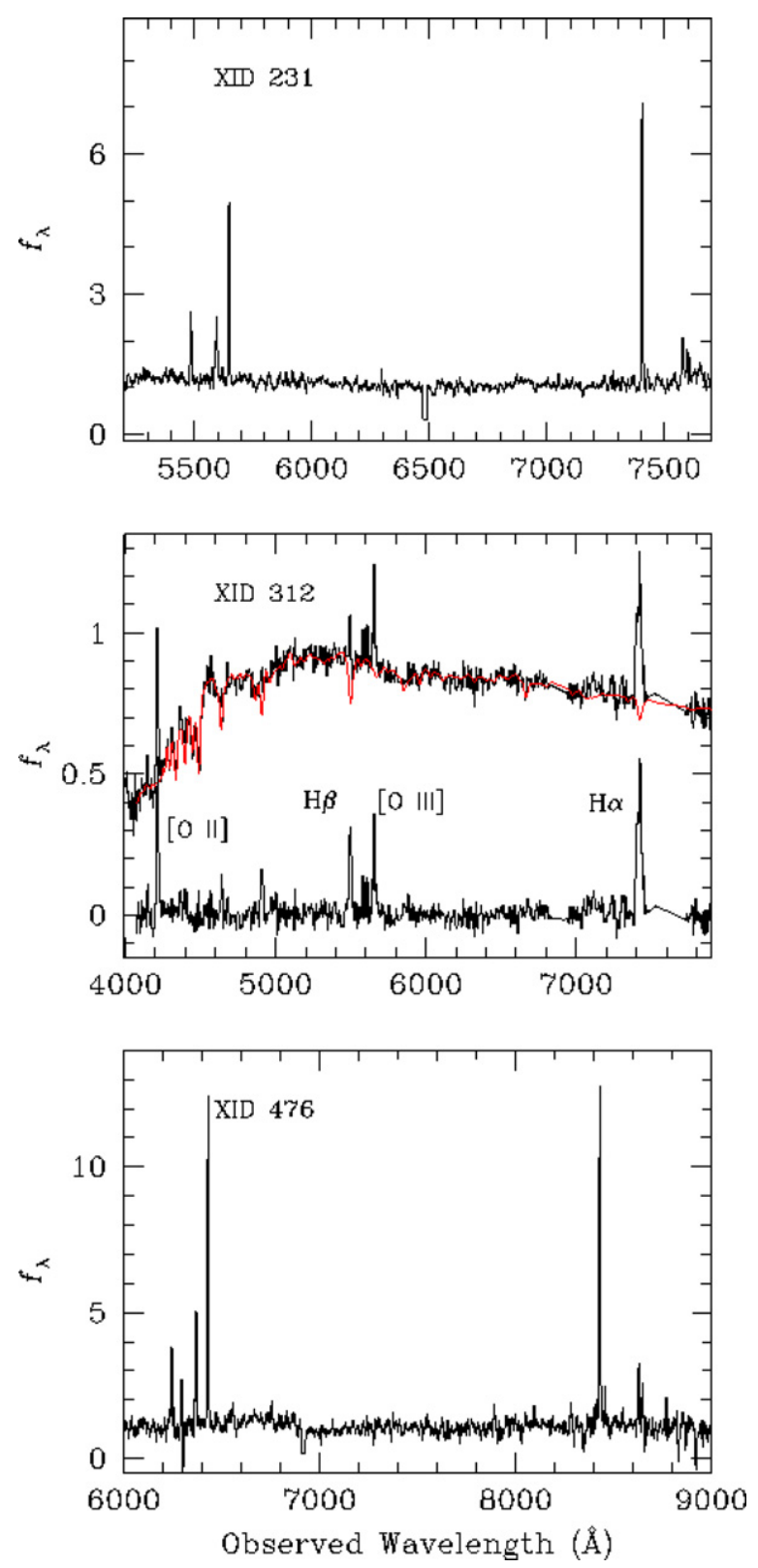

Figure 3. Optical spectra taken with either Keck/DEIMOS (XID $=231$ and 476) or VLT/FORS2 (XID = 312). Units are given in relative flux. For XID-312 we plot the spectrum before and after stellar continuum subtraction. The best-fit continuum is shown in red. 


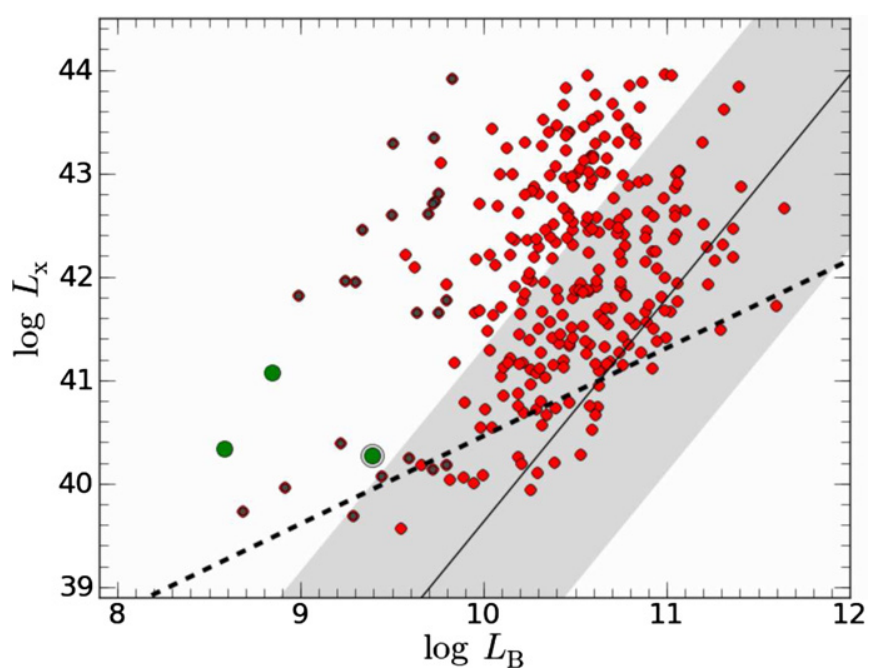

Figure 4. X-ray luminosity vs. $B$-band luminosity for $\mathrm{X}$-ray sources in the E-CDF-S (Lehmer et al. 2005) covered by HST with $L_{X}<10^{44} \mathrm{erg} \mathrm{s}^{-1}$ and $z_{\text {spec }}<1.0$. Three X-ray sources associated with galaxies having $z<0.3$ are shown in green. Sources with stellar masses below $M_{*}<3 \times 10^{9} M_{\odot}$ have additional gray circles. The solid line indicates where normal elliptical galaxies/star-forming typically lie (O'Sullivan et al. 2003). The dashed line shows the $1 \sigma$ upper bound for star-forming galaxies from Lehmer et al. (2010). We have encircled the position of XID-312 as the variable source showing a broad $\mathrm{H} \alpha$ line.

the typical relation for early-type galaxies, including the three galaxies at $z<0.3$ that are the focus of this study.

Now we turn to the optical line emission; see Figure 3 for additional clues as to the nature of these sources. We use traditional emission-line ratio diagnostics (e.g., Kewley et al. 2006) to determine the dominant source of photo-ionization in these galaxies (AGN activity or star formation). We perform a line-fitting routine to measure the strengths of the emission lines. We first fit the spectrum with a low-order polynomial to model the continuum adjacent to the emission line and then subtract it from the spectrum. In the cases of XID-476 and XID-231, we do not detect any starlight in the continuum. Line fluxes are measured by integrating our best fit obtained for a multi-Gaussian model. For XID-312, there is significant galaxy continuum, and so we use GANDALF (Sarzi et al. 2006) with BC03 models (Bruzual \& Charlot 2003) to model and subtract the galaxy continuum before applying the same line-fitting routines. In case of XID-476 and XID-231, a single Gaussian is sufficient to model the line profiles, but for XID-312 with its broad component in $\mathrm{H} \alpha$ and line blending with [N II], a multicomponent fit is necessary. We scale the spectra to the flux in the broad ACS $F 606 W$ filter.

In Figure 5, we plot the line ratios for $[\mathrm{O} \mathrm{III]} / \mathrm{H} \beta$ versus $[\mathrm{NII}] / \mathrm{H} \alpha$. We find that the line ratios are not typical of Seyfert 2 galaxies; all fall below the demarcation line of Kewley et al. (2006) that cleanly separates star-forming and AGNdominated galaxies. Two of the three galaxies are close to the demarcation line of Kauffmann et al. (2003) that was established to include galaxies having a composite nature (AGN + star formation). For comparison, the locations of low-mass Seyfert 2 galaxies, as described in Barth et al. (2008), are indicated by small blue circles; these galaxies have been selected to be bona fide AGNs by their emission-line ratios. Two of our three galaxies are located at low $[\mathrm{N} \mathrm{II}] / \mathrm{H} \alpha$ and high $[\mathrm{O}$ III $] / \mathrm{H} \beta$. Given the low stellar masses of our sample, these low ratios are most naturally explained as a metallicity effect (Tremonti et al. 2004; Groves et al. 2006; Ludwig et al. 2012; Stern \& Laor 2012).

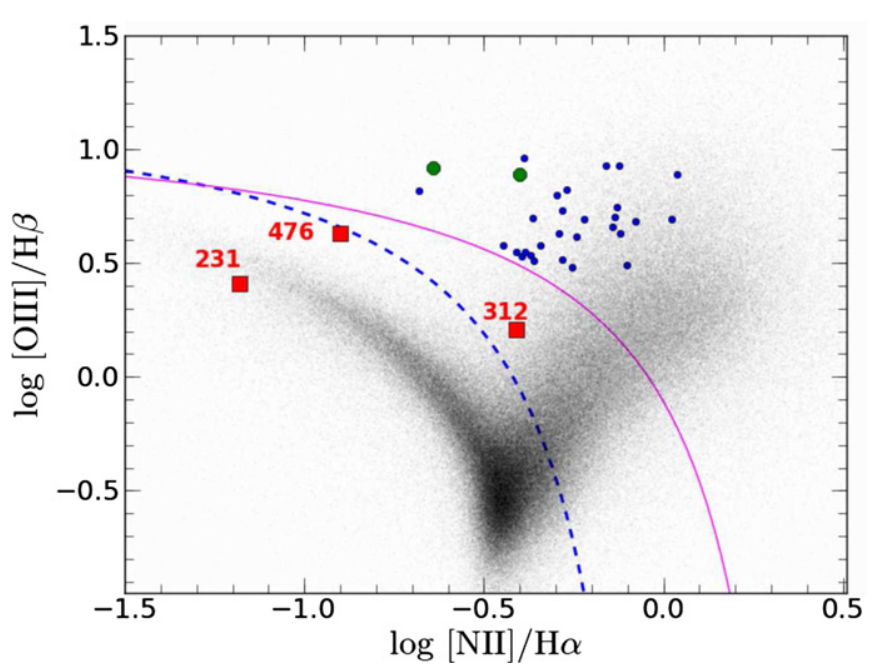

Figure 5. Line-ratio diagram for [O III] 5007/H $\alpha$ vs. [N II] 6583/H $\alpha$. NGC 4395 and POX 52 are shown as green circles. The blue circles mark the positions of low-mass Seyfert II galaxies from Barth et al. (2008). The solid curve is the "maximum starburst" line from Kewley et al. (2006), and the dashed line is from Kauffmann et al. (2003).

From the $[\mathrm{N} \mathrm{II}] / \mathrm{H} \alpha$ ratio, using the empirical calibration of Nagao et al. (2006), we estimate the metallicities of the three host galaxies to be in the range of $0.3-0.6$ solar metallicity. From the total emission-line intensity ratios $I(\mathrm{H} \alpha) / I(\mathrm{H} \beta)$, we estimate that all three galaxies show internal extinction ranging from $A_{V}=0.9$ to $1.6 \mathrm{mag}$.

In the case of XID-312, we report here a possible identification of a broad $\mathrm{H} \alpha$ line in the low-resolution optical spectrum from Szokoly et al. (2004). We performed the decomposition of the $\mathrm{H} \alpha$ profile into a Gaussian for the broad component and the [O III] line profile to model the narrow $\mathrm{H} \alpha$ and [N $\mathrm{NI}]$. We estimate that the broad component has an FWHM of $\sim 1400 \mathrm{~km} \mathrm{~s}^{-1}$ (corrected for instrumental resolution) but caution that the resolution of this spectrum is low, and the continuum is dominated by galaxy light. The corresponding $\mathrm{BH}$ mass would be $\log M_{\mathrm{BH} / M_{\odot}}=5.3$ using the calibration of Greene \& Ho (2005). Although X-ray variability of this source and the broad $\mathrm{H} \alpha$ line are strong indications of an $\mathrm{AGN}$, the overall line ratios place this galaxy in the composite $(\mathrm{SF}+\mathrm{AGN})$ region in the BPT diagram (Stern \& Laor 2012; see Figure 5).

We can use the narrow line emission as a further test of the origin of the X-ray emission. We take the total $\mathrm{H} \alpha$ luminosity as an upper limit to the SFR, under the conservative assumption that there is no contribution to the line emission from an AGN. SFRs are corrected for Galactic and internal extinction based on the measured Balmer decrement assuming a Calzetti (2000) extinction curve. Using $\mathrm{H} \alpha$ luminosity, we find that the upper limits to the SFRs are $\sim 0.2-0.6 M_{\odot} \mathrm{yr}^{-1}$ and are consistent with those seen in galaxies with masses of $\sim 10^{9} M_{\odot}$ (Elbaz et al. 2007). As a second SFR estimator, we use the extinctioncorrected GALEX-NUV flux measurement, which gives a consistent SFR estimation again assuming no contribution from accretion. We find that for these upper limits on the SFRs, the X-ray emission is $>4 \sigma$ (XID-231, XID-476) and $\sim 3 \sigma$ (XID-312) larger than expected for star-forming galaxies (see Figure 6) using the relations given in Mineo et al. (2011) and Lehmer et al. (2010).

Therefore, there are two possible explanations for our sources. First, they have one or several highly super-Eddington X-ray binaries, or ultra-luminous X-ray sources. Second, they contain accreting nuclear BHs. In either case, they are interesting 


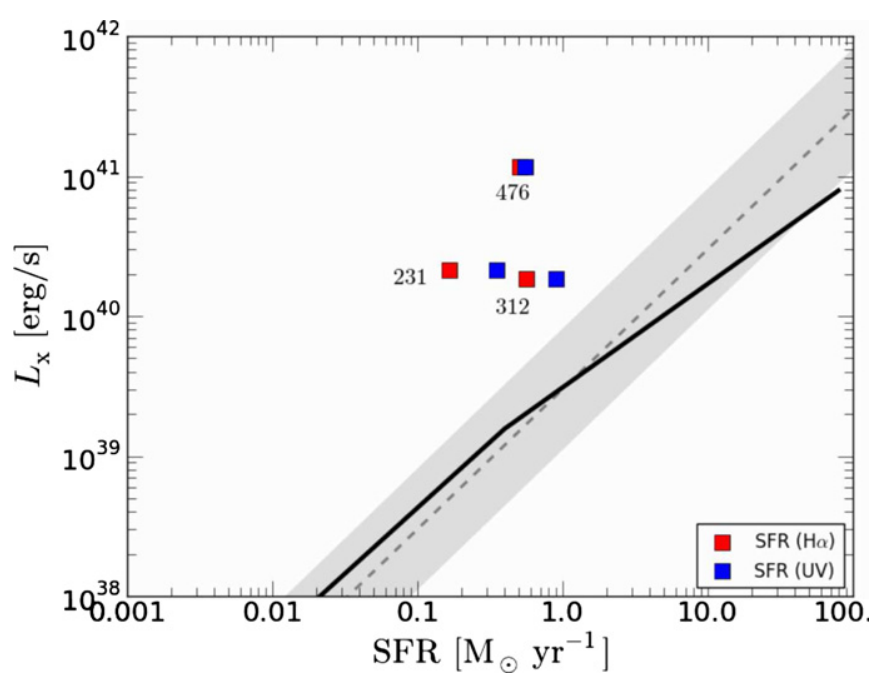

Figure 6. X-ray luminosity vs. star formation rate estimated from $\mathrm{H} \alpha$ luminosity (red) and UV continuum measured from the GALEX-NUV photometry. We make the assumption that all measured flux is due to star formation and no contribution is due to the presence of an AGN. This includes the possible contamination of scattered light to the measured UV continuum flux. The dashed line shows the best fit for local galaxies by Mineo et al. (2011) with a scatter of 0.4 dex marked as the shaded area. The solid line shows the relation from Lehmer et al. (2010).

outliers from the SFR $-L_{\mathrm{X}}$ relations. In the case of XID-312, the X-ray variability and broad $\mathrm{H} \alpha$ line both suggest that the source is powered by a massive $\mathrm{BH}$. We test the possibility of some super-Eddington X-ray binaries through a simple Monte Carlo test. We find that the probability for the two remaining galaxies to be outliers $(>4 \sigma)$ in the $L_{X}-$ SFR relation is $<0.2 \%$ based on our parent population of $\sim 2100$ galaxies at $z<0.3$. We expect at most one false positive detection at $4 \sigma$ within our parent. For this test we make the conservative assumption that we have a uniform X-ray sensitivity of the 4 Ms survey for the full parent population.

\section{HOST-GALAXY MORPHOLOGY}

We examine the host morphologies of each AGN candidate to provide some clues as to the formation mechanism of these lowmass BHs and their hosts. XID-312 falls within the GOODSSouth area with coverage with five $H S T$ filter bands (ACS: $F 435 W, F 606 W, F 775 W, F 850 L P$, and $F 814 W$ ). We could not find a point source in a two-dimensional fit to the $H S T$ images (given a limiting host-to-nuclear flux ratio host/nuclear $>50$ ) that included an empirical point-spread function (PSF) model created from nearby stars. We use a composition of three Sérsic profiles to fit the galaxy of XID-312. We use an $n=1$ and $n=4$ model to describe the disk and the bulge of the galaxy and an extended component with $n=0.42$. We estimate the bulge-tototal ratio $B / T=0.16$ (in the $z$ band) showing that the galaxy is mostly disk dominated. Although the galaxy is disk dominated, we do not see any signs of spiral arms, even at the resolution of $H S T$. For the estimated BH mass of $2 \times 10^{5} M_{\odot}$ from the broad $\mathrm{H} \alpha$ emission line and the stellar bulge mass, we find for the measured $B / T$, XID-312 falls onto the BH mass-bulge mass relation established for local galaxies by Haering \& Rix (2004, see Figure 7). The bulge component of XID-312 appears to be consistent with the relation at higher mass as well as the bulge components of disks from Greene et al. (2008) with similar BH masses.

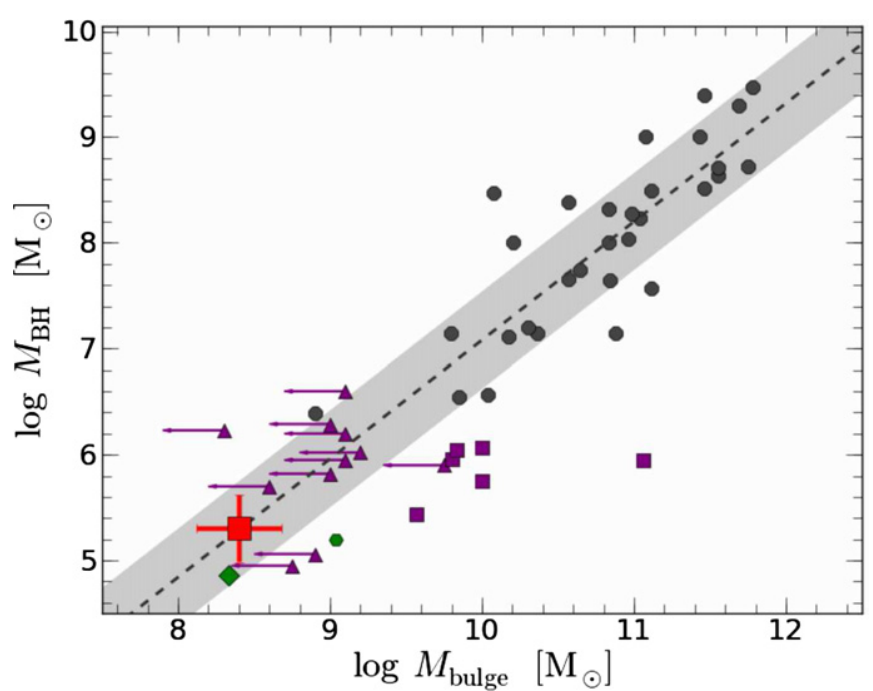

Figure 7. $M_{\mathrm{BH}}$ as a function of bulge mass. The estimate for XID-312 is shown as a red solid square. We overplot the local inactive sample from Haering \& Rix (2004) (gray symbols) as well as their best fit (dashed line) and a typical 0.3 dex uncertainty (shaded area). The low-mass BH sample from Greene et al. (2008) is shown as purple symbols. The solid squares mark compact smooth objects (likely to be ellipticals or spheroidals), and the filled triangles indicate the bulge components of disk galaxies. We use the smaller sample from Greene et al. (2008) rather than the Jiang et al. (2011b) sample because Greene et al. convert to stellar mass in a way that is more directly comparable with our measurements. We add the bulge mass estimate for POX 52 (green hexagon) and the total mass estimate for NGC 4395 (green diamond).

The morphological analysis of XID-476 using GALFIT to fit a Sérsic model shows that its host galaxy is an early-type galaxy based on the best-fit Sérsic index $\left(n_{\text {Sersic,F850LP }}=3.3\right)$. We do not find a point source (host/nuclear $>40$ ) in the center of the galaxy. Since we do not have a broad emission line to use to estimate the $\mathrm{BH}$ mass, we convert the stellar bulge mass into a BH mass using the relation from Haering \& Rix (2004), yielding a $\mathrm{BH}$ mass of $2.5 \times 10^{5} M_{\odot}$.

XID-231 has the lowest stellar mass of all three candidates $\left(\log M_{*}=8.3\right)$ and shows the most peculiar host among all three galaxies. We find no sign of a point source (host/nuclear $>40$ ) from our two-dimensional imaging analysis. Although the bestfit single Sérsic index is $n=2.4$, the residuals are significant and suggest that a single Sérsic model is insufficient to describe the host morphology. Adding further Sérsic models to the fit reduces the residuals, but we cannot identify a prominent bulge or disk component even by fixing some of the parameters. We conclude that this galaxy is of an irregular type. We estimate a $\mathrm{BH}$ mass of $1.5 \times 10^{5} M_{\odot}$ based on the total estimated stellar mass, given the morphological irregularity. The Sérsic indices together with the stellar masses and X-ray properties of the galaxies are listed in Table 1.

Finally, we make use of the different HST filter bands and look at the radial color profiles of the host galaxies. The analysis of XID-312 reveals a blue core with an $F 606 W-F 850 L P$ color gradient of $0.5 \mathrm{mag}$ (see Figure 8 ). The $F 435 W-F 606 W$ color gradient is even steeper $(1.25 \mathrm{mag})$ between the center and a radius of $\sim 0.7 \mathrm{kpc}$. It may be that the blue light is scattered from an AGN (see, e.g., Zakamska et al. 2005). In the case of XID-476, we find a similar blue core as for XID-312. The $F 606 W-F 850 L P$ color shows a steep gradient of 0.7 mag toward the center starting at a similar radius of about $0.7 \mathrm{kpc}$ (see Figure 8). Although the $F 606 W-F 850 L P$ color gradient of XID-231 shows a slightly bluer color ( $0.25 \mathrm{mag}$; see Figure 8$)$ 

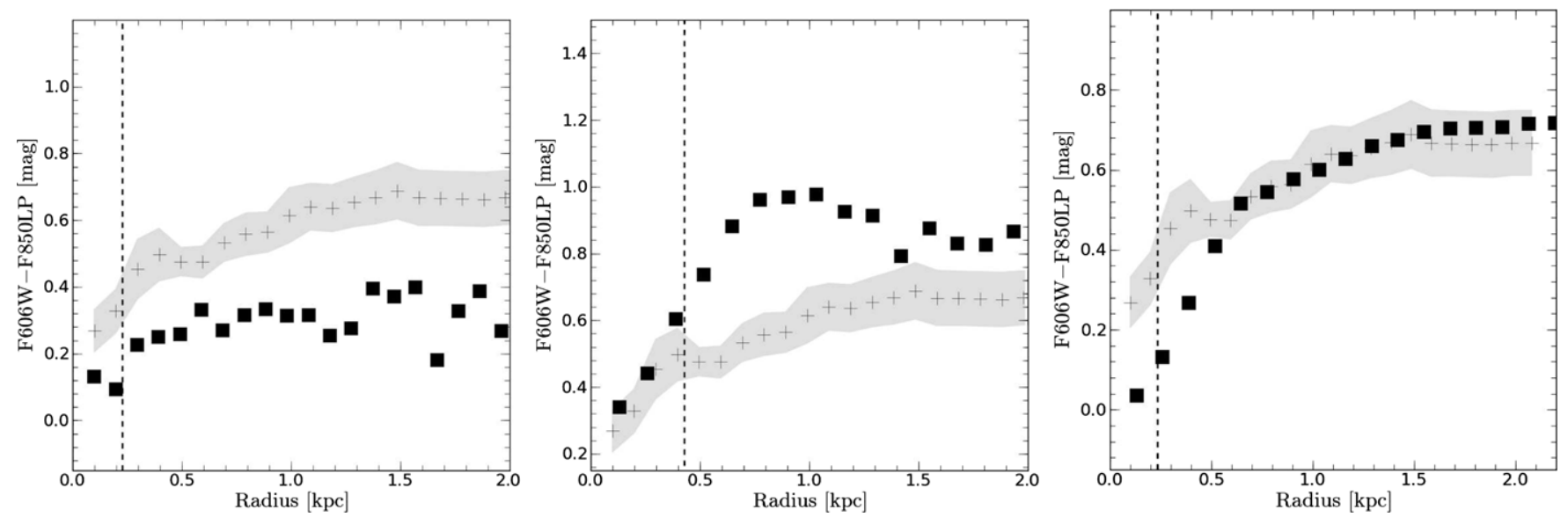

Figure 8. Observed $F 606 W-F 850 L P$ color profile of (from left to right) XID-231, XID-476, and XID-312 (solid squares). For comparison we show the average color profile of a redshift and mass matched sample of inactive galaxies (crosses) and the error of the mean as the gray shaded area. The vertical line shows the typical size of the PSF.

Table 1

AGNs in Low-mass Galaxies

\begin{tabular}{lcccccccccc}
\hline \hline ID & R.A. & Decl. & Redshift & $R_{\mathrm{AB}}$ & $\log M_{*}{ }^{\mathrm{a}}$ & $n_{\text {Sersic }}$ & X-Ray Counts & $\log f_{X}{ }^{\mathrm{b}}$ & $\log L_{X}{ }^{\mathrm{b}}$ & $\log M_{\mathrm{BH}}$ \\
\hline $231^{\mathrm{c}}$ & 52.98483 & -27.736667 & 0.128 & 22.7 & $8.3(7.7)$ & 2.4 & $9.4_{-3.4}^{+4.8}$ & -15.3 & 40.3 & 5.2 \\
$476^{\mathrm{c}}$ & 53.207625 & -28.006750 & 0.285 & 23.6 & $8.5(8.2)$ & 3.3 & $8.8_{-3.7}^{+5.0}$ & -15.3 & 41.1 & 5.4 \\
$312^{\mathrm{d}}$ & 53.094917 & -27.873389 & 0.131 & 20.4 & $9.2(8.8)$ & 2.9 & $112.4_{-13.9}^{+15.1}$ & -15.4 & 40.3 & 5.3 (virial) \\
\hline
\end{tabular}

Notes.

a Mass estimate based on GEMS (Rix et al. 2004), while that in parentheses is from the MUSYC survey (Cardamone et al. 2010).

b $0.5-8.0 \mathrm{keV}$ band; rest-frame $L_{X}$.

c X-ray ID given in Lehmer et al. (2005).

d X-ray ID given in Xue et al. (2011); XID = 521 in Giacconi et al. (2002) and Szokoly et al. (2004).

toward the center, the overall color profile remains flat. We compare the observed color gradients of our candidates with an average color profile of a redshift and mass matched sample $\left(\sim 10^{9} M_{\odot}\right)$ of inactive galaxies shown as a gray shaded profile in Figure 8. At $r<0.7 \mathrm{kpc}$ the color gradient of XID-312 and XID-476 is much steeper than the gradient of the comparison sample resulting in a color difference of $\sim 0.3 \mathrm{mag}$. We interpret this as evidence for a contribution from the AGN. The color gradients of these two sources also differ from the color profiles observed in point-source free unobscured X-ray-selected AGN host galaxies at $0.5<z<1.5$ (Ammons et al. 2011). This might reflect the complexity in disentangling AGN and SF activity on small scales. The overall profile of XID-231 is comparable with the average profile of inactive galaxies although the color is overall bluer.

\section{CONCLUSIONS}

We have identified three galaxies at $z<0.3$ with stellar masses below $3 \times 10^{9} M_{\odot}$ that likely harbor low-mass $\mathrm{BHs}$. If confirmed, these would be among the lowest-mass galaxies known to host an AGN. They are roughly an order of magnitude more luminous in the X-rays than expected for highly superEddington X-ray binaries, or ultra-luminous X-ray sources based on their continuum luminosities or their estimated SFRs. Assuming the empirical relation between $\mathrm{BH}$ mass and stellar bulge mass (e.g., Haering \& Rix 2004), we can estimate the BH masses to be of order $\sim 2 \times 10^{5} M_{\odot}$.

Accreting BHs in low-mass galaxies may contribute significantly to the hard X-ray background (Xue et al. 2012) so studying the host-galaxy properties in detail is an important task. The morphological analysis of the HST imaging data shows that each host is different. While XID-476 has an early-type host galaxy, XID-312 shows an intermediate and XID-231 an irregular host galaxy. Although all three galaxies show different morphologies, XID-312 and XID-476 have in common a blue core in the center of the galaxy $(<0.7 \mathrm{kpc})$. This suggests that the ionizing source is located in the center of the galaxy and could therefore be an AGN. The diversity in the host morphology provides an insight into how BHs interacted with their host environment in the early universe (Reines et al. 2011; Jeon et al. 2012).

Of the three low-mass AGN candidates, XID-312 shows the strongest evidence of having an accreting BH. Besides the excess in the X-ray emission (compared to SFR and optical continuum luminosity) and the X-ray variability (Young et al. 2012), this object shows an indication of a broad $\mathrm{H} \alpha$ emission line with an FWHM $=1400 \mathrm{~km} \mathrm{~s}^{-1}$ resulting in a corresponding $\mathrm{BH}$ mass of $\sim 2 \times 10^{5} \mathrm{M}_{\odot}$. This estimate is consistent with the $\mathrm{BH}$ mass prediction using the local BH mass-bulge mass. Although only a single object with independent $\mathrm{BH}$ and bulge mass estimate, XID-312 nicely extends the local relation at the low-mass end. For the other two sources the strong observed X-ray emission (although based on typically $<10$ X-ray counts), more than $4 \sigma$ above the expected emission from star-forming galaxies, is the strongest argument for hosting an AGN. Performing a conservative Monte Carlo test, we find that the probability for just being outliers as starforming galaxies is $<0.2 \%$ based on our parent population of $\sim 2100$ galaxies at $z<0.3$. More definitive proof that these two galaxies harbor AGNs could come from additional epochs of X-ray observations looking for variability similar to XID-312. In addition, resolved spectroscopy of the core region of the host 
looking for notable line ratios might also give further evidence of the presence of an AGN.

Eventually we want to understand whether all low-mass galaxies, such as those studied in this paper, host supermassive BHs. However, the story is complicated because as one goes to lower stellar mass, both the total occupation fraction (number of galaxies hosting a BH (e.g., Volonteri \& Natarajan 2009; Bellovary et al. 2010) and the distribution of Eddington ratios (e.g., Schulze \& Wisotzki 2010; Nobuta et al. 2012; Aird et al. 2012) may change in principle. Without independent constraints on the Eddington ratio distribution, we can derive only crude limits on the occupation fraction (e.g., Greene 2012). However, we hope to gain more insights, and better statistics, from studying the full sample of 27 potential low-mass BHs residing in low-mass galaxies identified in this work.

The authors fully appreciate the useful discussions with Aaron Barth and Masayuki Tanaka that improved the paper. This work was supported by the World Premier International Research Center Initiative (WPI Initiative), MEXT, Japan. Y.Q.X. acknowledges the financial support of the Thousand Young Talents (QingNianQianRen) program (KJ2030220004), the USTC startup funding (ZC9850290195), and the National Natural Science Foundation of China through NSFC-11243008. W. N. Brandt and B. Luo acknowledge the NASA ADP grant NNX10AC99G and the CXC grant AR3-14015X.

\section{REFERENCES}

Aird, J., Coil, A. L., Moustakas, J., et al. 2012, ApJ, 746, 90

Ammons, S. M., Rosario, D. J. V., Koo, D. C., et al. 2011, ApJ, 740, 3

Araya Salvo, C., Mathur, S., Ghosh, H., Fiore, F., \& Ferrarese, L. 2012, ApJ, 757,179

Babić, A., Miller, L., Jarvis, M. J., et al. 2007, A\&A, 474, 755

Barth, A. J., Greene, J. E., \& Ho, L. C. 2005, ApJL, 619, 151

Barth, A. J., Greene, J. E., \& Ho, L. C. 2008, AJ, 136, 1179

Barth, A. J., Ho, L. C., Rutledge, R. E., \& Sargent, W. L. W. 2004, ApJ, 607, 90

Bell, E. F., McIntosh, D. H., Katz, N., \& Weinberg, M. D. 2003, ApJS, 149, 289 Bellovary, J. M., Governato, F., Quinn, T. R., et al. 2010, ApJL, 721, L148

Boroson, B., Kim, D.-W., \& Fabbiano, G. 2011, ApJ, 729, 12

Bromm, V., \& Yoshida, N. 2011, ARA\&A, 49, 373

Bruzual, G., \& Charlot, S. 2011, MNRAS, 344, 1000

Calzetti, D., Armus, L., Bohlin, R. C., et al. 2000, ApJ, 533, 682

Cardamone, C. N., van Dokkum, P. G., Urry, C. M., et al. 2010, ApJS, 189, 270

Cooper, M., Yan, R., Dickinson, M., et al. 2012, MNRAS, 425, 2116

Desroches, L.-B., Greene, J., \& Ho, L. C. 2009, ApJ, 698, 1515

Desroches, L.-B., \& Ho, L. C. 2009, ApJ, 690, 267

Devecchi, B., \& Volonteri, M. 2009, ApJ, 694, 302

Dewangan, G. C., Mathur, S., Griffiths, R. E., \& Rao, A. R. 2008, ApJ, 689, 762

Dong, X.-B., Ho, L. C., Yuan, W., et al. 2012, ApJ, 755, 167

Dong, X.-B., Wang, T., Yuan, W., et al. 2007, ApJ, 657, 700

Elbaz, D., Daddi, E., Le Borgne, D., et al. 2007, A\&A, 468, 33

Fabbiano, G. 2006, ARA\&A, 44, 323

Fan, X., Narayanan, V. K., Lupton, R. H., et al. 2001, AJ, 122, 2833

Filippenko, A. V., \& Ho, L. C. 2003, ApJL, 588, L13

Filippenko, A. V., \& Sargent, W. L. W. 1989, ApJL, 342, L11

Gallo, E., Treu, T., Marshall, P. J., et al. 2010, ApJ, 714, 25
Giacconi, R., Zirm, A., Wang, J., et al. 2002, ApJS, 139, 369

Greene, J. E. 2012, NatCo, 3, 1304

Greene, J. E., \& Ho, L. C. 2004, ApJ, 610, 722

Greene, J. E., \& Ho, L. C. 2005, ApJ, 630, 122

Greene, J. E., \& Ho, L. C. 2006, ApJL, 641, L21

Greene, J. E., \& Ho, L. C. 2007a, ApJ, 656, 84

Greene, J. E., \& Ho, L. C. 2007b, ApJ, 667, 131

Greene, J. E., Ho, L. C., \& Barth, A. J. 2008, ApJ, 688, 159

Groves, B. A., Heckman, T. M., \& Kauffmann, G. 2008, MNRAS, 371, 1559

Haering, N., \& Rix, H.-W. 2004, ApJL, 604, L89

Ho, L. C., Filippenko, A. V., \& Sargent, W. L. W. 1997, ApJ, 487, 568

Ho, L. C., Kim, M., \& Terashima, Y. 2012, ApJ, 759, 16

Jeon, M., Pawlik, A. H., Greif, T. H., et al. 2012, ApJ, 754, 34

Jiang, Y.-F., Greene, J. E., \& Ho, L. C. 2011, ApJ, 737, 45

Jiang, Y.-F., Greene, J. E., Ho, L. C., Xiao, T., \& Barth, A. J. 2011, ApJ, 742, 68

Kauffmann, G., Heckman, T. M., Tremonti, C., et al. 2003, MNRAS, 346, 1055

Kewley, L. J., Groves, B., Kauffmann, G., \& Heckman, T. 2006, MNRAS, 372, 961

Koushiappas, S. M., Bullock, J. S., \& Dekel, A. 2004, MNRAS, 354, 292

Lehmer, B. D., Alexander, D. M., Bauer, F. E., et al. 2010, ApJ, 724, 559

Lehmer, B. D., Brandt, W. N., Alexander, D. M., et al. 2005, ApJS, 161, 21

Leipski, C., Gallo, E., Treu, T., et al. 2012, ApJ, 744, 152

Li, Y., Hernquist, L., Robertson, B., et al. 2007, ApJL, 665, 187

Ludwig, R. R., Greene, J. E., Barth, A. J., \& Ho, L. C. 2012, ApJL, 756, L51

Mineo, S., Gilfanov, M., \& Sunyaev, R. 2011, AN, 332, 349

Miniutti, G., Piconcelli, E., Bianchi, S., Vignali, C., \& Bozzo, E. 2010, MNRAS, 401, 1315

Nagao, T., Maiolino, R., \& Marconi, A. 2006, A\&A, 459, 85

Nobuta, K., Akiyama, M., Ueda, Y., et al. 2012, ApJ, 761, 143

Ohkubo, T., Nomoto, K., Umeda, H., Yoshida, N., \& Tsuruta, S. 2009, ApJ, 706, 1184

O’Sullivan, E., Ponman, T. J., \& Collins, R. S. 2003, MNRAS, 340, 1375

Peterson, B. M., Bentz, M. C., Desroches, L.-B., et al. 2005, ApJ, 632, 799

Popesso, P., Dickinson, M., Nonino, M., et al. 2009, A\&A, 494, 443

Ranalli, P., Comastri, A., \& Setti, G. 2003, A\&A, 399, 39

Reines, A. E., \& Deller, A. T. 2011, ApJL, 750, L24

Reines, A. E., Sivakoff, G. R., Johnson, K. E., \& Brogan, C. L. 2011, Natur, 470, 66

Rix, H.-W., Barden, M., Beckwith, S. V. W., et al. 2004, ApJS, 152, 163

Sarzi, M., Falcón-Barroso, J., Davies, R. L., et al. 2006, MNRAS, 366, 1151

Satyapal, S., Böker, T., Mcalpine, W., et al. 2009, ApJ, 704, 439

Satyapal, S., Vega, D., Heckman, T., O’Halloran, B., \& Dudik, R. 2007, ApJL, 663, L9

Schulze, A., \& Wisotzki, L. 2010, A\&A, 516, 87

Secrest, N. J., Satyapal, S., Gliozzi, M., et al. 2012, ApJ, 753, 38

Shields, J. C., Rix, H.-W., Sarzi, M., et al. 2007, ApJ, 654, 125

Silverman, J. D., Green, P. J., Barkhouse, W. A., et al. 2005, ApJ, 618, 123

Silverman, J. D., Mainieri, V., Salvato, M., et al. 2010, ApJS, 191, 124

Stern, J., \& Laor, A. 2012, MNRAS, 426, 2703

Szokoly, G., Bergeron, J., Hasinger, G., et al. 2004, ApJS, 155, 271

Terashima, Y., Kamizasa, N., Awaki, H., Kubota, A., \& Ueda, Y. 2012, ApJ, 752,154

Treister, E., Virani, S., Gawiser, E., et al. 2009, ApJ, 693, 1713

Tremonti, C. A., Heckman, T. M., Kauffmann, G., et al. 2004, ApJ, 613, 898

Van Wassenhove, S., Volonteri, M., Mayer, L., et al. 2012, ApJL, 748, L7

Vanzella, E., Cristiani, S., Dickinson, M., et al. 2008, A\&A, 478, 83

Volonteri, M., \& Natarajan, P. 2009, MNRAS, 400, 1911

Wrobel, J. M., Greene, J. E., Ho, L. C., \& Ulvestad, J. S. 2008, ApJ, 686, 838

Xiao, T., Barth, A. J., Greene, J. E., et al. 2011, ApJ, 739, 28

Xue, Y. Q., Brandt, W. N., Luo, B., et al. 2010, ApJ, 720, 368

Xue, Y. Q., Luo, B., Brandt, W. N., et al. 2011, ApJS, 195, 10

Xue, Y. Q., Wang, S. X., Brandt, W. N., et al. 2012, ApJ, 758, 129

Young, M., Brandt, W. N., Xue, Y. Q., et al. 2010, ApJS, 187, 560

Young, M., Brandt, W. N., Xue, Y. Q., et al. 2012, ApJ, 748, 124

Zakamska, N. L., Schmidt, G. D., Smith, P. S., et al. 2005, AJ, 129, 1212 